\title{
Aplicações da Robótica Educacional para o Desenvolvimento do Pensamento Computacional no Contexto do Ensino Médio Integral
}

\author{
Isabelle M.L. Souza \\ Universidade Federal de Campina \\ Grande, Campina Grande, Paraíba \\ isabellelima@copin.ufcg.edu.br
}

\author{
Wilkerson L. Andrade \\ Universidade Federal de Campina \\ Grande, Campina Grande, Paraíba \\ wilkerson@computacao.ufcg.edu.br
}

\author{
Lívia M. R. Sampaio \\ Universidade Federal de Campina \\ Grande, Campina Grande, Paraíba \\ livia@computacao.ufcg.edu.br
}

\section{RESUMO}

O Pensamento Computacional (PC) é uma abordagem que pode favorecer o processo de resolver problemas. Nesse sentido, considerando a importância da habilidade de resolução de problemas para o ser humano no momento atual, é factível o investimento em práticas educacionais que impulsionem a introdução do PC na vivência de estudantes desde a Educação Básica (EB). Pesquisas na área de Educação em Computação dedicam esforços na proposição de orientações, instrumentos e tecnologias que suportem a introdução do PC em ambientes escolares da EB. A Robótica Educacional (RE) é vista como uma dessas tecnologias que se destaca por promover habilidades como: trabalho em equipe, raciocínio lógico e criatividade; habilidades estreitamente alinhadas à ideia do PC. No entanto, muitos estudos, sobretudo àqueles inseridos no contexto da EB, não buscam investigar o impacto que a RE pode proporcionar no desenvolvimento de estudantes, evidenciando, deste modo, a necessidade de investigações empíricas. O objetivo deste trabalho é investigar o impacto que a RE causa no desenvolvimento do PC e no aprendizado dos componentes curriculares do Ensino Médio (EM) Integral. Para isso, realizamos um estudo do tipo pesquisa-intervenção com estudantes e professores da $1^{\text {a }}$ Série do EM Integral que analisou aspectos quantitativos e qualitativos. Os resultados obtidos indicam que a introdução da RE no EM Integral pode favorecer estudantes no desenvolvimento das habilidades do PC e no aprendizado dos componentes curriculares.

\section{CCS CONCEPTS}

- Computer systems organization $\rightarrow$ Embedded systems; Redundancy; Robotics; • Networks $\rightarrow$ Network reliability.

\section{PALAVRAS-CHAVE}

Robótica Educacional, Pensamento Computacional, Ensino Médio Integral.

\section{INTRODUÇÃO}

O Pensamento Computacional (PC) é uma forma de resolver problemas aplicando habilidades baseadas nos conceitos da Ciência da

Fica permitido ao(s) autor(es) ou a terceiros a reprodução ou distribuição, em parte ou no todo, do material extraído dessa obra, de forma verbatim, adaptada ou remixada, bem como a criação ou produção a partir do conteúdo dessa obra, para fins não comerciais, desde que sejam atribuídos os devidos créditos à criação original, sob os termos da licença CC BY-NC 4.0.

EduComp'21, Abril 27-30, 2021, Jataí, Goiás, Brasil (On-line)

(c) 2021 Copyright mantido pelo(s) autor(es). Direitos de publicação licenciados à Sociedade Brasileira de Computação (SBC).
Computação. Wing [27] menciona que é essencial trabalhar o PC desde o início do desenvolvimento cognitivo do ser humano. No entanto, ainda não existe uma definição formal para o PC, assim como orientações de como integrá-lo à Educação Básica (EB).

Instituições brasileiras têm difundido a cultura do PC com o intuito de promover as habilidades de resolução de problemas, como a Sociedade Brasileira de Computação ${ }^{1}$ que especifica o PC através de eixos essenciais do conhecimento da Ciência da Computação [19]. Além disso, o Currículo de Referência em Tecnologia e Computação ${ }^{2}$, propõe incluir disciplinas de tecnologia e informática no currículo da EB [8]. Essas iniciativas destacam a importância da inserção de recursos tecnológicos no âmbito do EB e oferecem orientações de como trabalhá-los em sala de aula.

Nesse cenário, a Robótica Educacional (RE) é vista como uma abordagem multidisciplinar que envolve o projeto, a montagem e o uso de robôs com base nos princípios da Engenharia, Computação, Matemática e Física. No entanto, a RE tem sido intensamente utilizada para o ensino de Ciências, Tecnologia, Engenharia e Matemática (STEM) e Ciência da Computação em diferentes níveis [21]. A inserção da RE na educação pode favorecer o processo de aprendizagem, uma vez que atividades lúdicas podem estimular o PC, contribuindo com o engajamento dos estudantes em STEM e, consequentemente, melhorando as habilidades de resolução de problemas dos estudantes [7].

O objetivo deste estudo foi estimular o desenvolvimento do PC no decorrer de atividades com RE no contexto da $1^{\text {a }}$ Série do Ensino Médio (EM) Integral, além de identificar suas relações com o aprendizado das ciências do currículo. Duas questões de pesquisa orientam o estudo: (Q1) Qual o impacto que a introdução da RE no EM Integral causa no desenvolvimento do PC? (Q2) Qual o impacto que a introdução da RE no EM Integral causa no aprendizado dos componentes curriculares? Para isso, realizamos um estudo longitudinal baseado no método de pesquisa-intervenção, considerando cinco fases: 1) Preparação; 2) Aplicação das intervenções da Robótica Autodescoberta; 3) Aplicação das intervenções da Robótica Fundamentada em Computação; 4) Aplicação de um survey com os professores participantes; 5) Análise dos efeitos que as intervenções causaram nos estudantes participantes. A amostra do estudo foi composta por 38 estudantes e 15 professores do $1^{\text {a }}$ Série do EM Integral do estado da Paraíba do ano letivo de 2018. Os estudantes foram organizados em dois grupos: experimental e controle. O primeiro grupo foi submetido às atividades com $\mathrm{RE}$, o segundo não. $\mathrm{O}$ desenvolvimento do PC foi avaliado por meio da prova do Bebras e

\footnotetext{
${ }^{1}$ Sociedade Brasileira de Computação: https://www.sbc.org.br

${ }^{2}$ Currículo de Referência em Tecnologia e Computação: http://curriculo.cieb.net.br
} 
o desempenho obtido nos componentes curriculares do EM Integral foi considerado para observar o desenvolvimento acadêmico dos estudantes.

Os dados apontam que o grupo experimental obtive desempenho superior ao grupo de controle na Prova do Bebras aplicada após a Robótica Autodescoberta, atingindo uma diferença de $36,16 \%$, demonstrando que a RE pode ajudar o desenvolvimento do PC em estudantes do EM Integral. O percentual de estudantes do grupo experimental que apresentam nos agrumamentos dos componentes curriculares uma média superior em relação ao grupo controle, varia entre 6,17\% a 15,86\% na Robótica Autodescoberta, entre 4,4\% a $22,42 \%$ na Robótica Fundamenta em Computação, e por fim, entre $0,0 \%$ a $13,68 \%$ no desempenho final do estudantes.

Este artigo está organizado da seguinte forma: A seção 2 descreve os principais conceitos que fundamentam o estudo; a seção 3 é dedicada a trabalhos relacionados; a seção 4 detalha o método aplicado, questões e hipóteses pesquisa, método de coleta e análise de dados; a seção 5 , apresenta os resultados, discussões e ameaças à validade; a seção 6, detalha as principais lições aprendidas; e finalmente, as conclusões e trabalhos futuros são apresentados na Seção 7.

\section{FUNDAMENTAÇÃO TEÓRICA}

\subsection{Pensamento Computacional}

PC está relacionado ao desenvolvimento cognitivo do raciocínio lógico aplicando habilidades computacionais [2]. Segundo Wing [27], o PC envolve habilidades essenciais para todos, não apenas a os cientistas da computação, argumentando a favor da inclusão dessas habilidades desde o início do desenvolvimento cognitivo das crianças. Na literatura, ainda não existe uma definição formal para o PC, apesar disso, alguns pesquisadores comumente o classifica através de conceitos e habilidades. Para [5], o PC visa desenvolver diversas habilidades como produtividade, inventividade e criatividade, e sugere que ele pode ser aplicado de duas formas: abstrair o problema e programar um computador para que ele possa resolver o problema mais rapidamente. Para Wing [27], as características do PC podem ser agrupadas em seis linhas, a saber: está mais relacionado a conceituar e não a programar, é fundamental e não utilitário, refere-se ao modo como os humanos pensam e não os computadores pensam, integra matemática e engenharia, diz respeito à construção de ideias e não à construção de produtos, e é para qualquer pessoa em qualquer lugar.

\subsection{Robótica Educacional}

A necessidade de novas práticas de ensino e aprendizagem que permitam aos estudantes adquirir e manter conhecimentos é uma temática que vem sendo muito discutida. Tal necessidade justifica o uso de tecnologias no ambiente educacional, como softwares, jogos e robótica. De acordo com Schons el al.[22], as tecnologias educacionais, se bem aplicadas, podem ajudar no desenvolvimento cognitivo de estudantes. Nos últimos anos, a RE tem sido considerada um recurso de aprendizagem que favorece o aprendizado, tanto das disciplinas STEM quanto em Ciência da Computação.

A RE, considerada uma evolução do LOGO de Papert [23], e pode favorecer a integração de conhecimentos científicas à simulação, permitindo que o estudante formule suas hipóteses, as implemente, teste, observe e realize as mudanças que considerar necessárias para os objetivos do robô [17]. Segundo Souza et al.[24], as iniciativas de introduzir a RE no EM mais discutidas na literatura abrangem: ensino de programação, ensino de robótica como ciência, ensino interdisciplinar de ciências, desenvolvimento de PC, participação em torneios e olimpíadas. Embora a utilização das práticas educacionais com RE seja diversa, é possível identificar situações práticas envolvendo problemas, seja para ensinar STEM ou lógica de programação, onde o estudante está envolvido no "aprender fazendo".

\subsection{Ensino Médio Integral da Paraíba}

Em 2017, devido a uma reformulação no sistema socioeducativo da Paraíba, a educação em tempo integral foi implantada em escolas do estado com o objetivo de promover uma educação de ressocialização. Dessa forma, as Escolas Cidadãs Integrais (ECIs) foram apresentadas com propostas pedagógicas que envolvem, sobretudo, a realidade local de cada escola de forma integral (manhã e tarde). Com o foco no protagonismo, as ECIs posicionam o jovem como ator principal da aprendizagem no decurso de ações que estimulam a criatividade, a construção e a solidariedade do jovem frente ao mundo real.

Com uma carga horária de 5.535 h/aula o EM das ECIs mantém o estudante semanalmente por 45 horas na escola para a aplicação de uma matriz curricular organizada conforme as orientações da Base Nacional Curricular acrescida de componentes curriculares articulando o mundo acadêmico e às práticas sociais. Para a $1^{\text {a }}$ Série, a matriz curricular do EM Integral envolve 18 componentes curriculares, a saber: Língua Portuguesa, Arte, Educação Física, Matemática, Biologia, Química, História, Geografia, Filosofia, Sociologia e Parte Diversificada (Inglês, Espanhol, Projeto de Vida, Orientações de Estudos, Práticas Experimentais e Disciplinas Eletivas).

\subsection{Prova do Bebras}

Bebras $^{3}$ é uma comunidade internacional da área de Computação e Educação que busca disseminar o PC entre estudantes de diversas idades. A comunidade Bebras promove anualmente um workshop que reúne professores e pesquisadores com o objetivo de formular questões que explorem o PC [12] e que irão compor a prova do Bebras. As questões são escritas em inglês e ficam disponíveis para países que desejam participar do desafio organizem suas provas e apliquem em suas escolas. As questões do Bebras são relacionadas às habilidades de PC, mas não exige conhecimentos de programação para serem respondidas [12]. Embora o objetivo principal do Bebras seja estimular o interesse dos estudantes pela computação, várias iniciativas buscam aplicar a prova para verificar as habilidades de PC em diferentes contextos $[3,4,7,18]$.

\section{TRABALHOS RELACIONADOS}

Estudos sobre PC evidenciam sua importância para a EB, especialmente para o EM. Em Rodrigues et al. [20] foi analisado o efeito causado pelo PC no desempenho acadêmico de estudantes do EM e observado uma correlação entre as habilidades de programação e o desempenho dos estudantes no Exame Nacional de Ensino Médio. No estudo de Costa et al. [11], estudantes (menores de 15 anos) foram analisados quantitativamente com o objetivo de avaliar o impacto do PC em uma abordagem conjunta com a Matemática, revelando um resultado positivo nas habilidades de resolução de

${ }^{3}$ Bebras: https://www.bebras.org 
problemas dos estudantes. No mesmo sentido, o trabalho apresentado por Souza et al. [25] demonstrou que o ensino de robótica alinhada às habilidades de PC favorece positivamente o desempenho acadêmico de estudantes do EM.

Ao Considerar a literatura sobre RE é possível observar que os estudos, em sua pluralidade, visam simplificar o ensino da ciências e possibilitar o desenvolvimento de habilidades, como trabalho em equipe, raciocínio lógico e criatividade [1,16]. Em Chiazzese [7] et al., os autores analisaram o efeito da RE no desenvolvimento do PC de estudantes do Ensino Fundamental. Neste estudo, as habilidades do PC foram avaliadas através da Prova do Bebras, e os resultados indicaram que a RE pode favorecer o desenvolvimento do PC. Nosso estudo se assemelha ao de [7] et al. [7] no sentido de introduzir RE com o objetivo de promover o PC e, consequentemente, auxiliar os estudantes no desenvolvimento escolar, porém aplicamos nosso estudo no EM Integral. Além disso, também utilizamos o Bebras como instrumento de coleta de dados. No entanto, em nosso estudo, investigamos como introduzir a RE no ambiente escolar através de um estudo longitudinal durante seis meses, diferentemente da proposta apresentada por [7].

Os resultados apresentados em Chaudhary et al. [6] demonstraram que projetar, construir e programar robôs favorece o desempenho das habilidades computacionais e de raciocínio lógico de estudantes. Como em [6], consideramos em nossas aulas de RE conceitos básicos de habilidades de pensamento lógico e computacional; entretanto, não limitamos o ensino desses conceitos à programação de robôs. Exploramos a prática combinada com o ensino da teoria. Neste caso específico, trabalhamos com rotação de motores, sentido de rotações, combinação de botões, sensores e fontes de energia, sem necessariamente abordar programação de robôs.

Destacamos que os estudos citados não apresentam um projeto educacional definido para aplicação da RE em sala de aula. Alguns destacaram os objetivos e as etapas aplicadas, porém, não apresentam elementos como materiais pedagógicos, planos de aula e diretrizes metodológicas para a replicação das práticas apresentadas. Por fim, poucos estudos avaliam quantitativa e qualitativamente os efeitos que um RE causa no PC, bem como no desenvolvimento cognitivo de estudantes do EM. Essa limitação pode estar relacionada à falta de instrumentos validados que auxiliem na avaliação do PC.

\section{METODOLOGIA}

Este estudo seguiu o método de pesquisa-intervenção, pois envolveu a identificação de uma dificuldade real, apoio de professores, apresentação de soluções, proposição e execução de intervenções e ao final, julgamento dos resultados obtidos [10]. Nesta seção, apresentamos detalhadamente a metodologia do estudo.

\subsection{Design da Pesquisa}

Este estudo seguiu 5 fases: 1) Preparação; 2) Aplicação das intervenções da Robótica Autodescoberta; 3) Aplicação das intervenções da Robótica Fundamentada em Computação; 4) Aplicação de um survey com os professores participantes; 5) Análise dos efeitos que as intervenções causaram nos estudantes participantes.

Para atingir os objetivos deste estudo, definimos as seguintes questões e hipóteses de pesquisa:
Q1: Qual o impacto que a introdução da RE no EM Integral causa no desenvolvimento do PC?

- H1.0: Não há indícios de que a introdução da RE no EM Integral impacta o desenvolvimento do PC.

- H1.1: A introdução da RE no EM Integral impacta o desenvolvimento do PC.

Q2: Qual o impacto que a introdução da RE no EM Integral causa no aprendizado dos componentes curriculares?

- H2.0: Não há indícios de que a introdução da RE no EM Integral impacta o aprendizado dos componentes curriculares.

- H2.1: A introdução da RE no EM Integral impacta o aprendizado dos componentes curriculares.

O design é composto por dois grupos (controle e experimental) de estudantes com perfis semelhantes, onde a única característica que os distingue é um curso de robótica aplicada ao grupo experimental (ver Tabela 1). Algumas variáveis fazem parte deste design, a saber:

- Ensino Médio (EM): Ações executadas durante o ano letivo.

- Atividades com Robótica (R): Aulas de intervenção com RE, propostas e aplicadas no experimento do presente estudo.

- Desempenho Curricular (D): Desempenho dos estudantes nos componentes curriculares, considerado o principal instrumento avaliativo na $\mathrm{EB}$, sendo assim, uma variável representativamente confiável do desenvolvimento do estudante nos componentes curriculares da EB.

- Habilidades do PC (PC) Desempenho na prova do Bebras que explora as habilidades do PC.

\section{Tabela 1: Design da pesquisa}

\begin{tabular}{ccc}
\hline Grupos & Variável Independente & Variável Dependente \\
\hline Experimental & $\mathrm{EM}+\mathrm{R}$ & $\mathrm{D}_{1}$ e $\mathrm{PC}_{1}$ \\
Controle & $\mathrm{EM}$ & $\mathrm{D}_{2}$ e $\mathrm{PC}_{2}$ \\
\hline
\end{tabular}

\subsection{Amostra e Coleta de Dados}

Os participantes deste estudo foram estudantes e professores da $1^{\text {a }}$ Série do EM Integral de uma escola estadual da Paraíba. O universo de estudantes estava distribuído em 5 turmas, por questões de capacidade instalada e disponibilidade de profissionais para a execução das intervenções, selecionamos, dentre elas, as 2 turmas com maior grau de homogeneidade.

Para a seleção, consideramos dados obtidos através do survey de perfil aplicado entre os dias 02 e 06 de abril de 2018 com os estudantes regularmente matriculados na $1^{\text {a }}$ Série do EM Integral que se faziam presentes no dia de sua aplicação. Dessa forma, selecionamos as 2 turmas que apresentaram menor diferença absoluta entre os seguintes critérios: quantidade de estudantes, média de acertos, quantidade de novatos e quantidade de repetentes.

Assim, consideramos uma amostra de 38 estudantes e 15 professores da $1^{\text {a }}$ Série do EM Integral. Para a análise, organizamos os estudantes em dois grupos (de controle e experimental) sendo a distribuição de estudantes de 47,4\% (18) do grupo de controle e 52,6\% (20) do grupo experimental (ver Tabela 2). O grupo de controle foi composto por estudantes da $1^{\text {a }}$ Série que não tiveram contato com $\mathrm{RE}$, já o grupo experimental, foi organizado por estudantes da $1^{\mathrm{a}}$ Série que tiveram contato com RE ao longo do ano letivo 2018. 
Tabela 2: Caracterização dos grupos de estudantes

\begin{tabular}{lcc}
\hline Turma & Amostra & Universo \\
\hline Turma 1 (Controle) & 18 & 27 \\
Turma 2 (Experimental) & 20 & 21 \\
Turma 3 & - & 40 \\
Turma 4 & - & 35 \\
Turma 5 & - & 32 \\
\hline Total & 38 & 152 \\
\hline
\end{tabular}

Buscando examinar o quanto a amostra utilizada representa a população de 152 estudantes da $1^{\text {a }}$ Série do EM Integral, aplicamos o cálculo amostral (1), onde $n=$ amostra calculada, $N=$ população, $Z$ $=$ variável normal padronizada associada ao nível de confiança, $p=$ verdadeira probabilidade do evento e $e=$ erro amostral. Verificamos que a amostra considerada representa a população de estudantes com erro amostral (diferença entre número estimado e número real) de $11,63 \%$ com $90 \%$ de confiança (probabilidade de que o erro amostral efetivo seja menor que o erro amostral admitido).

$$
n=\frac{N \cdot Z^{2} \cdot p \cdot(1-p)}{Z^{2} \cdot p \cdot(1-p)+e^{2} \cdot(N-1)}
$$

\subsection{Perfil dos Participantes}

Os grupos de estudantes (controle e experimental), possuem um perfil semelhante quanto ao sexo, idade média, número de repetência na $1^{\text {a }}$ Série e contato com a RE. Da amostra de 38 estudantes, $39,5 \%$ (15) são do sexo feminino e 60,5\% (23) do sexo masculino. A grande parte dos estudantes de ambos os grupos são do sexo masculino, $55,6 \%$ (10) no grupo de controle e $65 \%$ (13) no experimental.

A idade média da amostra de estudantes é de 15,6. O grupo de controle possui uma idade média de 15,8 enquanto que o experimental possuiu uma idade média de 15,5. 26,3\% (10) são de estudantes que estão repetindo a $1^{\text {a }}$ Série do EM e 73,7\% (28) são de estudantes que estão cursando-a pela primeira vez. $11,1 \%$ (2) dos estudantes do grupo de controle e 15\% (3) do grupo experimental nunca tiveram qualquer tipo de contato com RE e não demonstraram interesse no assunto; 83,3\% (15) dos estudantes do grupo de controle e $85 \%$ antes das atividades com RE, não haviam tido contato com RE, no entanto, possuíam interesse em aprender; por fim, 5,6\% (1) dos estudantes do grupo de controle já haviam tido algum tipo de contato com RE, no entanto, não demonstraram interesse no assunto.

A amostra de 15 professores representa $50 \%$ do universo de 30 professores que lecionaram na escola em 2018. Dos 15 professores, $26,7 \%$ (4) são do sexo masculino e 73,3\% (11) do sexo feminino. Em 2018, 20\% (3) dos professores lecionaram nos grupos de estudantes de controle e experimental, 2 componentes curriculares e $80 \%$ (12) lecionaram apenas 1. Com exceção de Projeto de Vida e Matemática, os componentes curriculares foram lecionados nos grupos de controle e experimental pelos mesmos professores.

No que se refere ao contato com RE, 60\% (9) dos professores nunca participaram de qualquer tipo de interação com a RE e $40 \%$ (6) participaram. Quanto ao conhecimento sobre os materiais de RE disponíveis na escola, 60\% (9) dos professores conhecem e $40 \%$ (6) não. Embora, os materiais sejam conhecidos por mais de $50 \%$ dos professores, 93,3\% (14) deles não utilizam os materiais como instrumento didático em sala de aula e apenas 6,7\% (1) utilizam. Por fim, $100 \%$ (15) demonstraram interesse em realizar um treinamento em RE.

\subsection{Atividades de Robótica}

Nosso curso de RE foi baseado nos conceitos de Construção $\mathrm{Pa}$ pert [23] e em metodologias de ensino RE comerciais como LEGO $^{\circledR}$ Education [28] e Fischertechnik $\mathrm{GmbH}^{\circledR}$ [15]. Organizamos as atividades em duas etapas: Robótica Autodescoberta e Robótica Baseada em Computação; cada uma com duração de 1 bimestre escolar. A primeira fase, executada durante o $2^{\circ}$ bimestre, contemplamos a promoção da autonomia do estudante na interação com materiais robóticos, já a segunda, executada durante o $3^{\circ}$ bimestre, envolvemos o aprendizado estruturado de robótica com base em fundamentos da computação. Descrevemos a seguir as especificidades de cada fase.

A Robótica Autodescoberta teve o objetivo de proporcionar aos estudantes conhecimentos de Ciências como Física, Matemática, Português, Tecnologia, e a criação de suas soluções robóticas para problemas da Ciência. O propósito destas atividades foi permitir que os estudantes trabalhassem com a RE de forma autônoma. A escolha dos conceitos das ciências foi norteada pelo programa de Física da $1^{\text {a }}$ serie do EM Integral programados para o $2^{\circ}$ bimestre escolar. Dessa forma, o professor de Física foi inserido no processo de planejamento sinalizando os conteúdos necessário para os estudantes.

As atividades contabilizaram uma carga-horária de 7 horas e seguiram a didática descrita a seguir:

(1) Contextualização: Apresentação da problemática, envolvendo os estudantes em discussões, buscando conhecimentos prévios dos estudantes, utilizando recursos audiovisuais, impressos, entre outros;

(2) Aprofundamento: Conexão dos conhecimentos das ciências com a atividade temática;

(3) Mão na massa: Construção livre de artefatos com o material de robótica que se relacionem aos conceitos discutidos.

As atividades utilizadas no Curso de Robótica Autodescoberta estão disponíveis online ${ }^{4}$.

Por sua vez, a Robótica Fundamentada em Computação buscou ensinar robótica baseada nos fundamentos da computação de forma a estimular as competências de PC através de exercícios práticos. Nesse contexto, o professor não é apenas um mediador do conhecimento conforme apresentado por Papert [23], mas também é responsável por "ensinar" conceitos que comumente não fazem parte do currículo do EM.

O conteúdo da Robótica Fundamentada em Computação foi norteada pelo programa do componente Lógica Matemática e de Programação da $1^{\text {a }}$ Série da modalidade Profissional Técnica de nível médio oferecido também nas escolas estaduais da Paraíba. Assim, tentamos seguir práticas que já são aplicadas no contexto educacional onde esta pesquisa foi aplicada.

Oito atividades foram planejadas para este curso com cargahorária equivalente a 14 horas no total. Essas aulas seguiram as etapas didáticas descritas a seguir:

\footnotetext{
${ }^{4}$ Repositório de estudos: https://bit.ly/2XC1tRn
} 
(1) Teórica: Ensino e discussão sobre conceitos técnicos de robótica e computação de forma unificada;

(2) Mão na massa: Partindo de uma situação problema, aplicação dos conceitos teóricos através da manipulação de materiais robóticos.

Para cada aula articulamos a união dos conceitos de Robótica e Computação buscando facilitar o entendimento de ambas as áreas e demonstrar que são conectáveis. Para os conteúdos de Robótica foram definidos: conceito de robô e robótica, aplicações da robótica, partes de um robô e Design de robô. Por sua vez, os conteúdos de computação foram: algoritmos, linguagem de programação (conceito), entrada e saída de dados, tipos de dados, variáveis, constantes, expressões lógicas e estruturas de repetição.

\subsection{Instrumentos}

Conduzimos a coleta de dados deste estudo longitudinalmente reunindo dados quantitativos e qualitativos relacionados ao desenvolvimento cognitivo do estudante durante as atividades de RE.

Utilizamos a prova do Bebras ${ }^{5}$ aplicada no Reino Unido em 2015 como um instrumento para medir o PC adquirido durante as atividades de RE. A prova contemplou 16 questões destinadas aos estudantes com idade acima de 13 anos. Atualmente, a prova não é realizado no Brasil, assim, traduzimos o prova para o português com a ajuda de outros pesquisadores do nosso grupo de pesquisa.

Consideramos as notas bimestrais e anuais do estudante na escola para representar o seu desempenho acadêmico ao longo da intervenção em 2018. Essas notas resultam da avaliação do professor nas respectivas disciplinas. Assim, os aspectos avaliados foram estipulados e aplicados pelos professores das disciplinas seguindo as normas do EM Integral.

Considerando os dados qualitativos utilizados neste estudo, tratase de aspectos relacionados ao progresso da aprendizagem dos estudantes em sala de aula, relatados por seus professores. Na observação de elementos qualitativos, é necessário manter contato substancial com os objetos e pessoas investigadas [13], consequentemente a experiência do professor com os estudantes, durante o ano letivo de 2018, foi essencial para atingir os objetivos deste estudo. Os instrumentos utilizados neste estudo estão disponíveis online $^{6}$

\subsection{Considerações Éticas}

Por envolver seres humanos, este estudo atendeu à resolução 196/96 do Conselho Nacional de Saúde do Brasil. Assim, registramos esta proposta de estudo na Plataforma Brasil $^{7}$ sob o número [Oculto devido à revisão cega]. Antes de iniciar as atividades estabelecidas, explicamos aos participantes sobre as ações que seriam realizadas cada participante leu e assinou o Termo de Consentimento Livre e Esclarecido para a realização deste estudo. Além disso, tratamos todos os dados de forma anônima. Todos os termos do Código de Ética deste estudo estão disponíveis online ${ }^{8}$.

\footnotetext{
${ }^{5}$ http://www.bebras.uk

${ }^{6}$ Repositório de estudos: https://bit.ly/2XC1tRn

${ }^{7}$ Plataforma Brasil: http://plataformabrasil.saude.gov.br/login.jsf

${ }^{8}$ Repositório de estudos: https://bit.ly/2XC1tRn
}

Tabela 3: Grupos de dados para análise

\begin{tabular}{ccc}
\hline Fase & $\begin{array}{c}\text { Pensamento Computacional } \\
\text { (Bebras) }\end{array}$ & $\begin{array}{c}\text { Componentes } \\
\text { Curriculares }\end{array}$ \\
\hline $\begin{array}{c}\text { Fase 01: Robótica } \\
\text { Autodescoberta }\end{array}$ & Pós-Teste I & Média do 2 Bimestre \\
Fase 02: Robótica & Pós-Teste II & Média do 3º Bimestre \\
$\begin{array}{c}\text { Fundamentada em Computação } \\
\text { Fase 03: Desempenho Final }\end{array}$ & Pós-Teste II & Média Anual \\
\hline
\end{tabular}

\section{RESULTADOS}

Para responder às questões de pesquisas, analisamos o desempenho dos estudantes dos grupos controle e experimental no teste do Bebras e nos componentes. Nesta seção, apresentamos os procedimentos de análise de dados e respondemos às questões de pesquisa.

\subsection{Procedimentos para Análise dos Dados}

Para a análise, agrupamos os componentes curriculares por área: Ciências Naturais (Biologia, Física e Química), Letras (Português, Arte, Espanhol e Inglês), Ciências Humanas (Filosofia, Geografia, História e Sociologia), Matemática e Parte Diversificada (Projeto de Vida, Orientação de Estudos, Disciplinas Eletivas e Práticas Experimentais). Posteriormente, associamos o desempenho acadêmico dos estudantes a cada um desses grupos. Por sua vez, o desempenho das habilidades de PC foi analisado com base na prova do Bebras. A Tabela 3 descreve as etapas consideradas no experimento e o tipo de métrica (Bebras ou desempenho acadêmico) aplicados na análise.

Ao analisar os pressupostos estatísticos de normalidade e homocedasticidade através dos testes de hipótese Shapiro-Wilk e Levene, respectivamente considerando um nível de significância $\alpha=0,05$ (ver Tabelas 4 e 5), rejeitamos com $95 \%$ de confiança a hipótese nula de que os dados seguem uma distribuição normal nos recortes do $2^{\circ}$ bimestre, $3^{\circ}$ bimestre e Média Anual de Ciências da Natureza; $2^{\circ}$ bimestre de Linguagens; $2^{\circ}$ bimestre, $3^{\circ}$ bimestre e Média Anual do agrupamento de Ciências Humanas; $2^{\circ}$ bimestre, $3^{\circ}$ bimestre de Matemática; $2^{\circ}$ bimestre da Parte Diversificada; assim como nos Pós-Teste 1 e Pós-Teste 2. Esses, apresentaram no teste Shapiro-Wilk um $p$-value resultante $>\alpha$.

Ainda com os dados apresentados nas Tabelas 4 e 5, consideramos que todos os recortes analisados tendem a heterogeneidade uma vez que no teste de Levene apresentaram um $p$-value resultante $>$ $\alpha$.

Como as premissas de normalidade e homocedasticidade não são atendidas, precisamos adotar testes estatísticos não paramétricos. Dessa forma, escolhemos o teste Mann-Whitney $U$ para avaliar se há diferença significativa entre o desempenho dos dois grupos (experimental e controle) no PC através do desempenho na prova do Bebras, bem como no o desempenho nos componentes curriculares. $\mathrm{O}$ índice de tamanho do efeito de Cohen foi usado para calcular e analisar o efeito do curso de robótica sob o desempenho nos componentes curriculares dos alunos [10]. Por fim, consideramos a correlação de Spearman $r$ para analisar a correlação entre a PC e o desempenho nos sujeitos [26].

Nos testes, usamos a linguagem de programação R e consideramos um nível de confiança de $95 \%$, uma significância estatística de $\alpha=0,05$ e o teste Mann-Whitney $U$. Além disso, os pressupostos estatísticos específicos do teste foram obedecidos. É importante 
Tabela 4: Pressupostos estatísticos: p-value dos testes de Normalidade e Homoscedasticidade para os recortes de análise dos agrupamentos

\begin{tabular}{ccccccc}
\hline \multirow{2}{*}{ Agrupamento } & \multicolumn{2}{c}{$2^{\circ}$ Bimestre } & \multicolumn{2}{c}{$3^{\circ}$ Bimestre } & \multicolumn{2}{c}{ Anual } \\
\cline { 2 - 7 } & Shapiro-Wilk & Levene & Shapiro-Wilk & Levene & Shapiro-Wilk & Levene \\
\hline Ciências da Natureza & 0,6213 & 3,68 & 0,6671 & 0,18 & 0,2715 & 1,28 \\
Linguagens & 0,1562 & 0,84 & $<0,05$ & 4,72 & $<0,05$ & 1,47 \\
Ciências Humanas & 0,8035 & 0,18 & 0,1429 & 0,09 & 0,1185 & 3,55 \\
Matemática & 0,09178 & 9,07 & 0,5441 & 2,68 & $<0,05$ & 0,092 \\
Parte Diversificada & 0,2328 & 4,32 & $<0,05$ & 2,62 & $<0,05$ & 0,39 \\
\hline
\end{tabular}

Tabela 5: Pressupostos estatísticos: p-value dos testes de Normalidade e Homoscedasticidade para os recortes de análise da Prova Bebras

\begin{tabular}{ccc}
\hline Teste & Pós-Teste 1 & Pós-Teste 2 \\
\hline Shapiro-Wilk & 0,1313 & 0,1409 \\
Levene & 4,02 & $<0,05$ \\
\hline
\end{tabular}

enfatizar que este teste aborda as condições variáveis da amostra de dados presente neste estudo.

\subsection{Q1: Qual o impacto que a introdução da RE no EM Integral causa no desenvolvimento do PC?}

Inicialmente, analisamos a média e o desvio padrão (DP) do desempenho dos alunos no Desafio Bebras aplicado após a conclusão de cada curso de robótica, denominado Fase 01 (Pós-Teste I) e Fase 02 (Pós-Teste II) conforme apresentado na Tabela 3. Verificando o desempenho de cada grupo (ver Tabela 6) observamos que o grupo experimental obteve um desempenho médio $36,16 \%$ melhor do que o grupo controle na Robótica Autodescoberta (Pós-Teste I). Em contra partida, no Robótica Fundamentada em Computação (Pós-Teste II), o desempenho dos estudantes do grupo experimental apresentou média inferior em relação ao grupo de controle de $-2,91 \%$.

Tabela 6: Média, desvio padrão e diferença média do desempenho dos grupos de estudantes no Bebras

\begin{tabular}{|c|c|c|c|c|c|}
\hline \multirow[t]{2}{*}{ Bebras } & \multicolumn{2}{|c|}{ Controle (C) } & \multicolumn{2}{|c|}{ Experimental (E) } & \multirow{2}{*}{$\begin{array}{l}\text { Diferença } \\
\text { Média (\%) }\end{array}$} \\
\hline & Média & $D P$ & Média & $D P$ & \\
\hline Pós-Teste I & 3,18 & 2,94 & 4,33 & 1,87 & 36,16 \\
\hline Pós-Teste II & 5,84 & 2,37 & 5,67 & 2,37 & $-2,91$ \\
\hline
\end{tabular}

Nesse sentido, buscando confirmar os indícios de que a RE não causa efeito no PC de estudantes do EM Integral aplicamos o Teste- $U$ de Mann-Whitney com um nível de confiança de $95 \%$ e significância $\alpha=0,05$. Os dados relacionados ao Teste- $U$ podem ser observados na Tabela 7, com os quais observamos o $p$-value $>\alpha$ para o PósTeste I e para o Pós-Teste II, assim, devemos aceitar a hipótese nula H1.0: Não há indícios de que a introdução da RE na $1^{a}$ Série do EM Integral impacta o desenvolvimento do PC; independente da metodologia (Robótica Autodescoberta ou Robótica Fundamentada em Computação).
Tabela 7: Teste de hipótese e tamanho do efeito da Prova Bebras

\begin{tabular}{|c|c|c|c|c|c|}
\hline Bebras & Teste- $U$ & p-value & Cohen d & $\begin{array}{c}\text { Tamanho do } \\
\text { Efeito }\end{array}$ & $(E>C) \%$ \\
\hline Pós-Teste I & 157,5 & 0,64890 & 0,21 & Pequeno & $58 \%$ \\
\hline Pós-Teste II & 114,5 & 0,93510 & 0,07 & Insignificante & $52 \%$ \\
\hline
\end{tabular}

Com os dados obtidos no Teste- $U$ não constatamos diferenças significativas no desempenho dos grupos experimental e de controle tanto no Pós-Teste I, quanto no Pós-Teste II, uma vez que para esses cenários $p$-value $>\alpha$, não sendo factível, dessa forma, afirmar que a Robótica Autodescoberta e Fundamentada em Computação são capazes de impactar significativamente às habilidades do PC. No entanto, ao analisarmos o tamanho do efeito do grupo experimental sobre o grupo de controle com o apoio do $d$ de Cohen [10], observamos um efeito positivo atribuído à Robótica Autodescoberta (Pós-Teste I) e à Robótica Fundamentada em Computação (Pós-Teste II). Em conformidade com a métrica de avaliação de Cohen [10], para os dados apresentados na Tabela 7, a Robótica Autodescoberta apresentou um efeito pequeno, já a Fundamentada em Computação demonstrou um efeito insignificante sobre as habilidade do PC.

Ainda observando a Tabela 7, como $d$ de Cohen é exatamente equivalente ao $Z$-score tabelado de uma distribuição normal padrão [9], a partir da consulta na tabela normal padrão verificamos que na fase da Robótica Autodescoberta (Pós-Teste I) o percentual dos estudantes do grupo experimental que apresentam uma média superior em relação ao grupo controle é de $58 \%$, já na fase Robótica Fundamenta em Computação (Pós-Teste II) o percentual é de $52 \%$.

Apesar do Teste- $U$ não ter oferecido as evidências necessárias para rejeitar a hipótese nula H1.0, o efeito da RE sobre as habilidades do PC no grupo experimental pode ser considerado positivo, visto que ao final de cada fase o grupo experimental apresentou uma média superior em relação ao grupo de controle. Além disso, existem, ao longo da intervenção, bons resultados relacionados à aprendizagem qualitativa dos estudantes envolvidos que demonstram a importância do PC para sua vivência. Consideramos que o trabalho destinado ao estímulo do PC pode ter auxiliado qualitativamente na evolução dos estudantes no sentido de resolver problemas aplicando os conceitos da computação trabalhados no âmbito das atividades com RE.

\subsection{Q2: Qual o impacto que a introdução da RE no EM Integral causa no aprendizado dos componentes curriculares?}

Analisamos a média e o desvio padrão do desempenho acadêmico dos alunos após os cursos de robótica. Nesse caso, o percentual de alunos do grupo experimental que apresentou desempenho superior ao do grupo controle varia entre $6,17 \%$ a $15,86 \%$ na Fase 01 , entre $4,4 \%$ a $22,42 \%$ na Fase 02 , e por fim, entre $0,0 \%$ a $13,68 \%$ no desempenho final do estudantes na Fase 03 (ver Tabela 8). Embora seja verificado, em algumas fases dos agrupamentos, que a diferença média é negativa, fato que indica melhor desempenho do grupo de controle, através dos dados positivos é possível observar indícios de que a RE, seja Autodescoberta ou Fundamentada em Computação, 
pode impactar no ensino dos componentes curriculares da $1^{\text {a }}$ Série do EM Integral.

Diante desses resultados, consideramos que a RE melhorou a aprendizagem dos alunos nos componentes curriculares analisados, tanto por meio da Robótica Autodescoberta quanto da Robótica Fundamentada em Computação. Assim, para refutar a hipótese nula H2.0: Não há indícios de que a robótica na $1^{\text {a }}$ Série do EM Integral favorece o ensino dos componentes curriculares; aplicamos o Teste- $U$ de Mann-Whitney com um nível de confiança de $95 \%$ e significância $\alpha=0,05$.

Tabela 8: Média, desvio padrão e diferença média do desempenho dos grupos de estudantes nos agrupamentos dos componentes curriculares

\begin{tabular}{ccccccc}
\hline \multirow{2}{*}{ Agrupamento } & \multirow{2}{*}{ Fase } & \multicolumn{2}{c}{ Controle (C) } & \multicolumn{2}{c}{ Experimental (E) } & \multirow{2}{*}{$\begin{array}{c}\text { Diferença } \\
\text { Média (\%) }\end{array}$} \\
\cline { 3 - 6 } & & Média & DP & Média & DP & \\
\hline Ciências da & Fase 01 & 5,36 & 0,950 & 6,21 & 1,32 & 15,86 \\
Natureza & Fase 02 & 5,13 & 0,890 & 6,28 & 1,53 & 22,42 \\
& Fase 03 & 5,41 & 0,910 & 6,15 & 1,31 & 13,68 \\
\hline Linguagens & Fase 01 & 7,38 & 0,550 & 7,12 & 0,67 & $-3,59$ \\
& Fase 02 & 6,87 & 0,830 & 7,20 & 1,43 & 4,80 \\
& Fase 03 & 7,26 & 0,410 & 7,30 & 0,92 & 0,51 \\
\hline Ciências & Fase 01 & 6,97 & 0,470 & 7,40 & 0,60 & 6,17 \\
Humanas & Fase 02 & 7,30 & 0,500 & 6,98 & 0,82 & $-4,38$ \\
& Fase 03 & 6,70 & 0,500 & 6,97 & 0,87 & 4,09 \\
\hline Matemática & Fase 01 & 6,75 & 1,780 & 5,11 & 2,33 & $-24,30$ \\
& Fase 02 & 6,01 & 1,640 & 3,78 & 2,70 & $-37,10$ \\
& Fase 03 & 5,37 & 1,500 & 5,42 & 1,90 & 0,97 \\
\hline Parte & Fase 01 & 7,60 & 0,590 & 7,50 & 0,2 & $-1,32$ \\
Diversificadan & Fase 02 & 7,05 & 0,450 & 7,36 & 0,53 & 4,40 \\
& Fase 03 & 7,41 & 0,360 & 7,41 & 0,32 & 0,00 \\
\hline
\end{tabular}

Os dados obtidos com o Teste- $U$ podem ser observados na Tabela 9, com eles, verificamos um $p$-value $<\alpha$ nos agrupamentos de Ciências da Natureza na Fase 01 (Robótica Autodescoberta) e Fase 02 (Robótica Fundamentada em Computação), em Linguagens na Fase 02, em Ciências Humanas na Fase 01 e na Parte Diversificada na Fase 02; dessa forma rejeitamos a hipótese nula H2.0: Não há indícios de que a introdução da RE no EM impacta o aprendizado dos componentes curriculares; e aceitamos a hipótese alternativa H2.1: A introdução da RE no EM impacta o aprendizado dos componentes curriculares.

É importante destacar que no componente curricular de Matemática, a Fase 01 e Fase 02 resultou $p$-value $<\alpha$, no entanto, esses fazem parte dos cenários em que a diferença média entre o desempenho dos grupos de controle e experimental é negativa, indicando que os estudantes do grupo de controle possuem melhor desempenho quando comparados ao grupo experimental. Essa realidade pode ser justificada por vários fatores, o principal, pode estar relacionado ao aspecto do componente curricular em cada grupo ser conduzido por professores distintos. Cada professor possui uma metodologia, estratégias de planejamento, aspectos avaliativos, formação docente, entre outras variáveis que podem refletir na condução do trabalho em sala de aula, sobretudo no ensino e avaliação, condições que refletem diretamente neste estudo, uma vez que, o desempenho construído pelo estudante ao longo do componente curricular foi considerado como referência para as análises.
Tabela 9: Teste de hipótese e tamanho do efeito nos agrupamentos dos Componentes Curriculares

\begin{tabular}{ccccccc}
\hline Agrupamento & Fase & Teste-U & $\boldsymbol{p}$-value & Cohen d & $\begin{array}{c}\text { Tamanho do } \\
\text { Efeito }\end{array}$ & (E > C) \% \\
\hline Ciências da & Fase 01 & 202,0 & $<0,05$ & 0,73 & Médio & $76 \%$ \\
Natureza & Fase 02 & 182,5 & $<0,05$ & 0,88 & Grande & $81 \%$ \\
& Fase 03 & 152,0 & 0,16720 & 0,60 & Médio & $72 \%$ \\
\hline \multirow{3}{*}{ Linguagens } & Fase 01 & 114,5 & 0,31690 & 0,42 & Pequeno & $66 \%$ \\
& Fase 02 & 166,5 & $<0,05$ & 0,27 & Pequeno & $60 \%$ \\
& Fase 03 & 153,0 & 0,15740 & 0,04 & Insignificante & $51 \%$ \\
\hline Ciências & Fase 01 & 218,0 & $<0,05$ & 0,79 & Médio & $78 \%$ \\
Humanas & Fase 02 & 87,0 & 0,23750 & 0,46 & Pequeno & $67 \%$ \\
& Fase 03 & 157,0 & 0,11380 & 0,36 & Pequeno & $64 \%$ \\
\hline \multirow{3}{*}{ Matemática } & Fase 01 & 77,0 & $<0,05$ & 0,78 & Médio & $78 \%$ \\
& Fase 02 & 50,0 & $<0,05$ & 0,96 & Grande & $83 \%$ \\
& Fase 03 & 111,0 & 0,82440 & 0,05 & Insignificante & $51 \%$ \\
\hline Parte & Fase 01 & 95,0 & 0,09128 & 0,21 & Pequeno & $58 \%$ \\
Diversificada & Fase 02 & 178,0 & $<0,05$ & 0,62 & Médio & $73 \%$ \\
& Fase 03 & 115,5 & 0,96800 & 0,03 & Insignificante & $51 \%$ \\
\hline
\end{tabular}

Com os dados obtidos no Teste- $U$ não observamos diferenças significativas entre o desempenho dos grupos de controle e experimental nos demais agrupamentos dos componentes curriculares uma vez que para esses cenários $p$-value $>\alpha$, não sendo factível, dessa forma, afirmar que a robótica, seja ela Autodescoberta ou Fundamentada em Computação, é capaz de impactar significativamente o ensino desses componentes curriculares. No entanto, ao analisar o tamanho do efeito do grupo experimental sobre o grupo de controle com o apoio do $d$ de Cohen [10] nesses cenários, observamos um efeito positivo atribuído à robótica, seja ela Autodescoberta (01 Fase) ou Fundamentada em Computação (02 Fase).

Em conformidade com a métrica de avaliação de Cohen [10], para os dados apresentados na Tabela 9, no agrupamento de Ciências da Natureza, a Robótica Autodescoberta causou um efeito médio, a Fundamentada em Computação um efeito grande e a inserção da robótica de uma forma geral causou no desempenho final dos estudantes um efeito médio. Em Linguagens, a Robótica Autodescoberta e Fundamentada em Computação causou um efeito pequeno. No agrupamento de Ciências Humanas, por sua vez, a Robótica Autodescoberta causou um efeito médio e a Robótica Fundamentada em Computação assim como a inserção da robótica de uma forma geral causou, no desempenho final dos estudantes, um efeito pequeno. Por fim, no agrupamento Parte Diversificada, a Robótica Autodescoberta causou um efeito pequeno e a Fundamentada em Computação um efeito médio. Não obstante, no agrupamento de Linguagem, Matemática e Parte diversificada o efeito causado pela $\mathrm{RE}$ sob o desempenho final dos estudantes é indiferente. O efeito da Robótica Autodescoberta e Fundamentada em Computação sobre o componente curricular de matemática não favorece o grupo experimental, uma vez que nesses cenários o grupo de controle se apresentou melhor que o grupo experimental.

Contudo, observamos que o impacto da RE é positivo e significativo no aprendizado dos componentes curriculares da $1^{\text {a }}$ Série do EM Integral no agrupamentos de Ciências da Natureza, Linguagens, Ciências Humanas e da Parte Diversificada, demonstrando que é possível melhorar o ensino e aprendizagem de conceitos das ciências quando a RE é inserida no cotidiano escolar. 


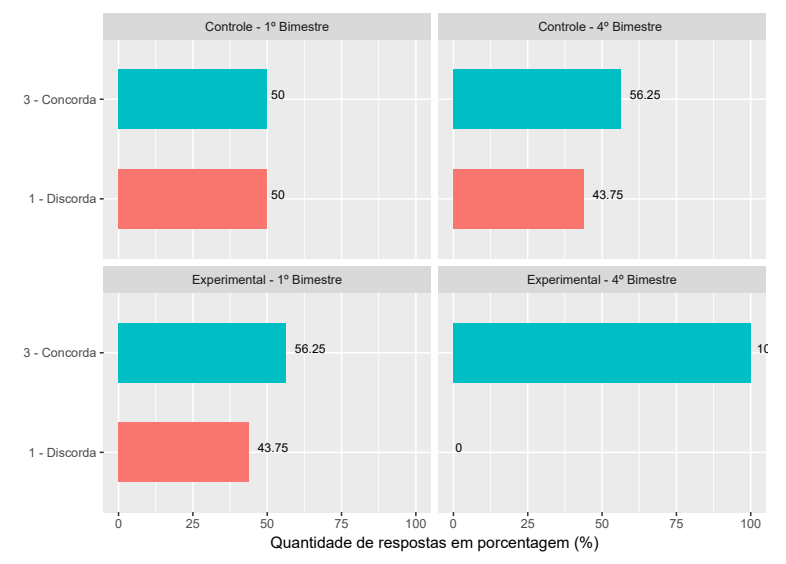

Figura 1: Os estudantes participaram efetivamente em meu componente curricular.

Ainda observando a Tabela 7, como $d$ de Cohen é exatamente equivalente ao $Z$-score tabelado de uma distribuição normal padrão [9], a partir da consulta na tabela normal padrão verificamos que na fase da Robótica Autodescoberta o percentual dos estudantes do grupo experimental que apresentam uma média superior em relação ao grupo controle varia entre $58 \%$ a $78 \%$, já na fase Robótica Fundamenta em Computação entre $60 \%$ a $81 \%$ e na média anual do estudantes entre $51 \%$ e $76 \%$.

Para complementar este estudo, consideramos ainda a interpretação de aspectos comportamentais dos estudantes realizada pelos professores ao longo do ano letivo de 2018. Questionamos os professores sobre participação, argumentação e aprendizagem dos estudantes dos grupos de controle e experimental através de um survey na escala Likert e construímos um comparativo entre os grupos de controle e experimental, antes ( $1^{\circ}$ bimestre) e após ( $4^{\circ}$ bimestre) a introdução da RE na vivência escolar dos mesmos, das seguintes afirmações:

- Os estudantes participaram efetivamente em minha disciplina. As atividade de participação podem ser realização das atividades, posicionamento em discussões em sala, assiduidade em aulas.

- A qualidade dos argumentos dos estudantes em discussões durante minhas aulas foi satisfatória. Os argumentos foram críticos, científicos e alinhados com os conteúdos estudados.

- Os estudantes demonstraram facilidade em aprender (independente dos resultados obtidos em atividades avaliativas) em minha disciplina.

Os dados obtidos apontam que os estudantes do grupo experimental, após as atividades com RE, aumentaram a participação em $43,75 \%$ dos componentes curriculares. Por sua vez, os estudantes do grupo de controle, aumentaram a participação em 6,24\% dos componentes curriculares (ver Figura 1).

No que se refere à qualidade dos argumentos dos estudantes, após as atividades com RE, os professores aumentaram a satisfação com a qualidade dos argumentos dos estudantes em seus componentes

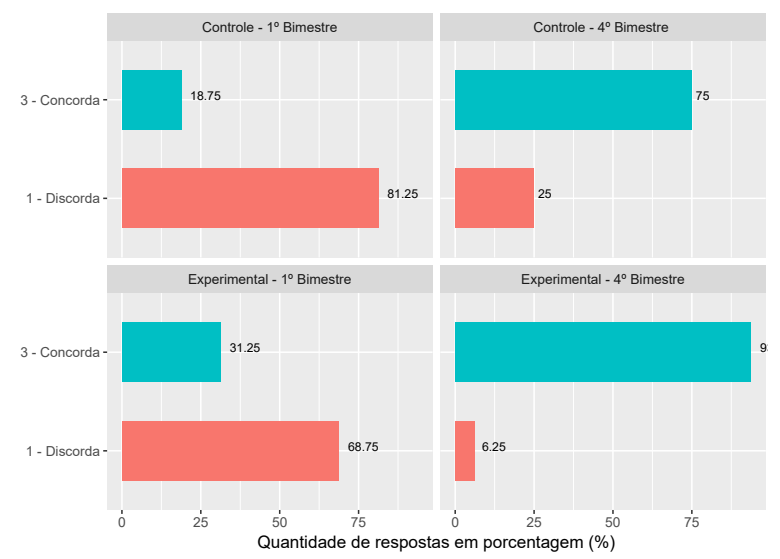

Figura 2: A qualidade dos argumentos dos estudantes em discussões durante meu componente curricular foi satisfatória.

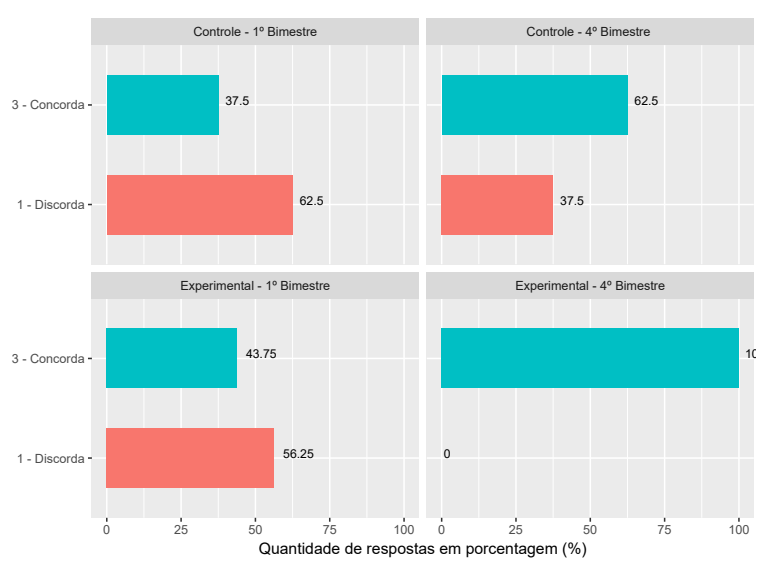

Figura 3: Os estudantes demonstram facilidade em aprender (independente dos resultados obtidos em atividades avaliativas).

curriculares em $62,5 \%$, enquanto no grupo de controle esse aumento correspondeu apenas a 56,25\% (ver Figura 2).

Visando entender o desenvolvimento dos estudantes de uma perspectiva observacional, questionamos os professores sobre a facilidade de aprendizagem dos estudantes, antes e após as atividades com RE. No grupo experimental, após as atividades com RE, a facilidade de aprendizagem foi verificada em $100 \%$ (16) dos componentes curriculares, enquanto no grupo de controle, foi em apenas $25 \%$ (ver Figura 3).

Embora o Teste- $U$ não tenha oferecido evidências para assegurar a significância da robótica em todas as fases dos agrupamentos, consideramos, com os dados apresentados, que as metodologias aplicadas neste estudo podem influenciar o desenvolvimento de estudantes da $1^{\text {a }}$ Série do EM Integral. Através dos dados referentes 
ao desempenho dos estudantes (Bebras e componentes curriculares) observamos que o grupo experimental em que a RE foi inserida, demonstra um desempenho superior ao grupo de controle que não teve contato com a RE. Em complemento, as observações obtidas com os professores corroboram com os dados obtidos nas análise, fortalecendo dessa forma a hipótese alternativa H2.1: A robótica na EB favorece o ensino dos componentes curriculares.

\subsection{Ameaças à Validade}

Este estudo possui ameaças à validade. Os surveys aplicados podem não ter coletado as informações desejadas. Além disso, a prova Bebras foi corrigida e catalogada manualmente, bem como os dados coletados nos surveys dos alunos e professores, e embora tenha sido aplicado a dupla checagem de dados, erros de correção atribuídos a fatores humanos podem ocorrer.

Neste estudo, não foi possível controlar fatores da vida escolar dos estudantes que podem influenciar os resultados, como dependência administrativa da escola, qualidade do ensino fundamental e atividades extracurriculares. Os estudantes podem ter se apropriado da prova do Bebras durante suas reaplicações, o que possivelmente ajudou a solucionar as dúvidas. Além disso, como eles responderam às mesmas perguntas, pode ter ocorrido desmotivação durante as reaplicações do Bebras.

As questões Bebras utilizadas no estudo foram traduzidas do inglês e, embora dois colaboradores externos tenham revisado o texto traduzido, ele podem conter viés de tradução e não representar adequadamente os objetivos das questões originais. Alguns elementos da prova do Bebras não são da realidade dos estudantes, o que pode ter influenciado a compreensão dos problemas. Além disso, não existe garantia de que o Bebras possa medir o PC. É importante ressaltar também que o desempenho acadêmico dos estudantes pode não representar a sua aprendizagem, principalmente nas disciplinas ministradas por professores diferentes nos grupos experimental e controle.

Por fim, a amostra analisada é representativa de estudantes da $1^{a}$ Série do EM Integral de uma escola pública da Paraíba, portanto não podemos generalizar os resultados alcançados para outras populações de alunos.

\section{LIÇÕES APRENDIDAS}

Nesta seção apresentamos as principais lições aprendidas neste estudo.

\subsection{Quanto aos Resultados Obtidos}

Os resultados relacionados ao desempenho dos estudantes nos componentes curriculares que apresentamos neste estudo, foram organizados em agrupamentos seguindo as orientações da BNCC e das matrize curricular da escola. Nessa circunstância, obtivemos confirmações estatísticas para confirmar que a RE pode ajudar no aprendizado dos agrupamentos dos componentes curriculares, no entanto, não verificamos as evidências em todos os agrupamentos.

Nessa conjuntura, buscando entender melhor os dados correlatos, estendemos os testes estatísticos dos agrupamentos para os componentes curriculares individualmente. Observamos que todos os agrupamentos que não resultaram em evidências positivas, existem componentes curriculares da BNCC com resultados positivos e significativos, como: Artes, Língua Portuguesa, Língua Espanhola, Língua Inglesa, Educação Física, Biologia, Sociologia, Geografia, Filosofia, História, Química, Física.

Desse modo, entendemos que o resultado real do impacto da $\mathrm{RE}$ sobre o aprendizado dos componentes curriculares pode ser superior aos resultados obtidos por agrupamentos, pois ao agrupar o desempenho dos componentes curriculares, os dados são balanceados e podemos camuflar impactos importantes. Além disso, é importante entender que cada componente curricular possui afinidades diferentes com a RE, ou seja, cada um pode ser impactado singularmente, com maior ou menor intensidade.

Acreditamos que as atividades com RE desenvolvidas com os estudantes, por terem sido de natureza prática, tenham auxiliado no processo de compreender e relacionar os conceitos estudados, nesses componentes curriculares, com situações do mundo real. Essa capacidade de relacionar conceitos teóricos com situações concretas, que é intimamente relacionada às habilidades do PC, pode auxiliar diretamente no aprender explicando, dessa forma, os resultados obtidos individualmente nos componentes curriculares citados.

\subsection{Quanto às Relações entre PC e os Componentes Curriculares}

De forma complementar, aplicamos o teste de Correlação de Spearman $r$ com o objetivo de analisar a correlação existente entre o desempenho em PC (Prova do Bebras) e dos componentes curriculares dos estudantes [26]. Com os dados da Tabela 10, observamos que no grupo experimental todas as fases dos agrupamentos possuem uma correlação positiva entre o desempenho em PC e o desempenho dos componentes curriculares. Enquanto isso, no grupo de controle, observamos nos agrupamentos de Ciências da Natureza e Linguagens correlações negativas, demonstrando que o desempenho dos estudantes em PC e nestes componentes curriculares, comumente, andam em sentidos opostos, situação diferente do grupo experimental em que o desempenho do PC tende a ser maior, em grande parte deles, além disso, o desempenho nos componentes curriculares também seguem a mesma tendência.

Seguindo as definições da intensidade da correlação de Spearman [26], no grupo experimental consideramos como muito fraca as correlações da Fase 01 de Ciências da Natureza e Matemática, assim como na Fase 02 da Parte Diversificada; como fraca as correlações da Fase 01 de Ciências da Natureza e Ciências Humanas, Fase 01 e Fase 02 de Linguagens, Fase 02 de Matemática e Fase 01 e Fase 03 da Parte Diversificada. Nos demais cenários consideramos as correlações como moderada.

Embora o objetivo principal deste estudo tenha sido entender como a RE impacta no desenvolvimento de estudantes do EM Integral, com os dados apresentados nesta seção, observamos que a relação entre o desempenho do PC e dos componentes curriculares é positiva para o grupo de estudantes que tiveram contato com RE como ferramenta de ensino. Além disso, os resultados da correlação corroboram com pesquisas presentes na literatura sobre PC [14, 20], bem como favorece o investimento de metodologias com RE para o ensino no EM. 
Tabela 10: Coeficientes de correlação de Spearman entre o desempenho na Prova Bebras e nos componentes curriculares

\begin{tabular}{cccc}
\hline Agrupamento & Fase & Controle (E) & Controle (E) \\
\hline Ciências da & Fase 01 & 0,61 & 0,09 \\
Natureza & Fase 02 & 0,22 & 0,41 \\
& Fase 03 & $-0,06$ & 0,61 \\
\hline \multirow{3}{*}{ Linguagens } & Fase 01 & 0,43 & 0,18 \\
& Fase 02 & $-0,10$ & 0,17 \\
& Fase 03 & $-0,04$ & 0,56 \\
\hline Ciências & Fase 01 & 0,35 & 0,27 \\
Humanas & Fase 02 & 0,20 & 0,70 \\
& Fase 03 & 0,07 & 0,63 \\
\hline \multirow{3}{*}{ Matemática } & Fase 01 & 0,46 & 0,07 \\
& Fase 02 & 0,25 & 0,50 \\
& Fase 03 & 0,24 & 0,60 \\
\hline Parte & Fase 01 & 0,35 & 0,30 \\
Diversificada & Fase 02 & 0,13 & 0,06 \\
& Fase 03 & 0,08 & 0,22 \\
\hline
\end{tabular}

\subsection{Quanto à Obrigatoriedade}

As atividades de intervenção não foram inseridas oficialmente no programa de curso dos estudantes e todos tinham o conhecimento de que os assuntos trabalhados não seriam utilizados nas avaliações da escola. Essa característica pode ter influenciado diretamente no interesse, participação e assiduidade dos estudantes da turma experimental durante as aulas de intervenções. Embora houvesse estudantes que demonstravam interesse por RE, a carência do compromisso por parte dos estudantes dificultou a realização das aulas. A chegada dos estudantes ao laboratório demandava tempo e, como consequência, o início das aulas era comprometido. No decorrer das atividades, identificamos estudantes dispersos, entrando e saindo espontaneamente da sala de aula, com expressões faciais negativas e de cansaço. É importante destacar que realizamos muitas das aulas nos últimos horários do dia, e como eles estavam inseridos no EM integral, é comum observar esgotamento do desempenho dos estudantes nesses horários.

A concretização do planejamento das aulas de intervenção era, em alguns momentos, prejudicadas. Por vezes, iniciamos uma aula com a retomada da atividade anterior para realizar o fechamento da aula, ou seja, a sequenciação didática das aulas era comprometida. Através das observações, podemos destacar avanço no progresso individual de alguns estudantes, no entanto, o mesmo não é possível afirmar quando olhamos para o grupo de forma coletiva. Assim é possível afirmar que exitem estudantes do grupo experimental que se empenharam nas atividades propostas, tal como nos demais componentes curriculares da $1^{\text {a }}$ Série, esses, qualitativamente avaliamos satisfatoriamente, no entanto, consideramos que o desempenho geral do grupo poderia ser melhor avaliado se as atividades tivessem sido incorporadas no processo de avaliação escolar.

Apesar dessas adversidades, consideramos os resultados obtidos como positivos, uma vez que é possível identificar diferença significativa entre o desempenho dos grupos experimental e de controle quando olhamos para o desempenho dos estudantes nos componentes curriculares. Entretanto, acreditamos que os resultados, tanto quantitativo como qualitativo, poderiam ser mais expressivos se a metodologia fizesse parte do projeto político pedagógico das ECIs da Paraíba.

\section{CONSIDERAÇÕES FINAIS}

Este estudo buscou estimular as habilidades do PC durante atividades com RE, bem como identificar suas relações com o aprendizado das ciências do currículo. Para isso, propomos intervenções com RE na $1^{\text {a }}$ Série do EM Integral, em uma ECI do governo da Paraíba, localizada na cidade de Campina Grande. Organizamos as intervenções em duas etapas intituladas de Robótica Autodescoberta e Robótica Fundamentada em Computação, onde aconteceram aulas, aplicações de survey e da prova do Bebras. Para a avaliar os efeitos da RE nas habilidade do PC, consideramos o desempenho dos estudantes na prova do Brebas, aplicada após cada fase das intervenções. Em se tratando dos efeitos que a RE causou no ensino dos componentes curriculares, consideramos os desempenhos bimestrais e anuais dos estudantes nos componentes curriculares.

Com os resultados obtidos, não é possível concluir estatisticamente que os estudantes que tiveram contato com as metodologias de ensino proposta, obtiveram um melhor desempenho na prova do Bebras aplicadas. No entanto, essa constatação pode estar relacionada às ameças internas, uma vez que os conteúdos trabalhados nas aulas de intervenções não faziam parte do conteúdo avaliado pela escola. Diante dessas ameaças à validade, que não puderam ser tratadas, consideramos que os resultados advindos da análise da primeira questão de pesquisa (Q1) são inconclusivos. Desse modo, destacamos como importante a realização de estudos complementares no cenário do EM Integral de forma a obter resultados com menos interferências.

Quanto aos componentes curriculares, os resultados obtidos demonstram que as metodologias aplicadas neste estudo podem influenciar o desenvolvimento de estudantes da EB, já que os estudantes que tiveram contato com a RE apresentaram um desempenho superior quando comparados aos que não tiveram contato com a RE, fatores que foram complementados com as observações dos professores. Não obstante, os resultados obtidos no componente curricular de Matemática, impossibilitam o cumprimento dos pressupostos de todos os recursos estatísticos utilizados, uma vez que, através da diferença média entre o desempenho dos grupos, observa-se que o grupo de controle possui desempenho superior quando comparado ao grupo experimental. Esse fato pode estar relacionado às ameaças internas de instrumentação, visto que em cada grupo o componente curricular foi conduzido por professores distintos, uma vez que consideramos o desempenho construído pelo estudante ao longo do componente curricular como referência para as análises.

\section{REFERÊNCIAS}

[1] Charoula Angeli and Nicos Valanides. 2020. Developing young children's computational thinking with educational robotics: An interaction effect between gender and scaffolding strategy. Computers in Human Behavior 105 (2020), 105954. https://doi.org/10.1016/j.chb.2019.03.018

[2] A. L. S. O. Araujo, W. L. Andrade, D. Guerrero, M. Melo, and I. M. L. Souza. 2018. Análise de Rede na Identificação de Habilidades Relacionadas ao Pensamento Computacional. In Brazilian Symposium on Computers in Education (Simpósio Brasileiro de Informática na Educação-SBIE). SBIE, Fortaleza, CE, BR, 655 
[3] A. L. S. O. Araujo, W. L. Andrade, D. D. S. Guerrero, and M. R. A. Melo. 2019. How Many Abilities Can We Measure in Computational Thinking?: A Study on Bebras Challenge. In Proceedings of the 50th ACM Technical Symposium on Computer Science Education (Minneapolis, MN, USA) (SIGCSE '19). ACM, New York, NY, USA, 545-551. https://doi.org/10.1145/3287324.3287405

[4] A. L. S. O. Araujo, J. S. Santos, W. L. Andrade, D. D. S. Guerrero, and V. Dagiene 2017. Exploring computational thinking assessment in introductory programming courses. In 2017 IEEE Frontiers in Education Conference (FIE). IEEE, Indianapolis, IN, USA, 1-9. https://doi.org/10.1109/FIE.2017.8190652

[5] P. Blikstein. Acesso em: 06 de Agosto de 2020. O pensamento computacional e a reinvenção do computador na educação. Disponivel em: https://goo.gl/YxKxvx (Acesso em: 06 de Agosto de 2020).

[6] V. Chaudhary, V. Agrawal, P. Sureka, and A. Sureka. 2016. An Experience Report on Teaching Programming and Computational Thinking to Elementary Level Children Using Lego Robotics Education Kit. In 2016 IEEE Eighth International Conference on Technology for Education (T4E). IEEE, Mumbai, India, 38-41.

[7] G. Chiazzese, M. Arrigo, A. Chifari, V. Lonati, and C. Tosto. 2018. Exploring the Effect of a Robotics Laboratory on Computational Thinking Skills in Primary School Children Using the Bebras Tasks. Proceedings of the Sixth International Conference on Technological Ecosystems for Enhancing Multiculturality TEEM'18 (2018), 25-30. https://doi.org/10.1145/3284179.3284186

[8] CIEB. 2018. Currículo de Referência em Tecnologia e Computação. In Currículo de Referência em Tecnologia e Computação. Centro de Inovação para a Educação Brasileira, CIEB, São Paulo-SP, 104. http://curriculo.cieb.net.br/assets/docs/ Curriculo_de_Referencia_em_Tecnologia_e_Computacao.pdf

[9] R. Coe. 2002. It's the effect size, stupid: What effect size is and why it is important. Semantic Scholar 1 (2002)

[10] L. Cohen, K. Morrison, and L. Manion. 2011. Research Methods in Education IEducation, Research methods. Routledge. n (2011), n.

[11] E. J. F. Costa, L. M. R. S. Campos, and D. D. S. Guerrero. 2017. Computational thinking in mathematics education: A joint approach to encourage problem-solving ability. In 2017 IEEE Frontiers in Education Conference (FIE). IEEE, Indianapolis, Indiana, USA, 1-8. https://doi.org/10.1109/FIE.2017.8190655

[12] V. Dagiene, S. Sentance, and G. Stupurienë-. 2017. Developing a TwoDimensional Categorization System for Educational Tasks in Informatics. Informatica 28 (01 2017), 23-44. https://doi.org/10.15388/Informatica.2017.119

[13] S. Easterbrook, J. Singer, M. Storey, and D. Damian. 2008. Selecting empirical methods for software engineering research. In Guide to advanced empirical software engineering. Springer, London, 285-311.

[14] E. J. C. Fidelis. 2017. Pensamento computacional na educação básica: uma abordagem para estimular a capacidade de resolução de problemas na matemática. UFCG 1,156 pages.

[15] $\mathrm{GmbH}^{\odot}$ Fischertechnik. 2018. Fischertechnik. Available in: https://www.fischertechnik.de/en 1 (2018).

[16] A. J. Hirst, J. Johnson, M. Petre, B. A. Price, and M. Richards. 2003. What is the best programming environment/language for teaching robotics using Lego Mindstorms? Artificial Life and Robotics 7, 3 (01 Sep 2003), 124-131. https: //doi.org/10.1007/BF02481160

[17] R. Maisonnette. 2002. A utilização dos recursos informatizados a partir de uma relação inventiva com a máquina: a robótica educativa. PROINFO-Programa Nacional de Informática na Educação, Curitiba-PR 1 (2002), 35.

[18] T. Palts and M. Pedaste. 2017. Tasks for Assessing Skills of Computational Thinking. In Proceedings of the 2017 ACM Conference on Innovation and Technology in Computer Science Education (Bologna, Italy) (ITiCSE '17). ACM, New York, NY, USA, 367-367. https://doi.org/10.1145/3059009.3072999

[19] A. Raabe. 2017. Referenciais de formação em computação: Educação básica. In Workshop sobre Educação em Computação, Sociedade Brasileira de Computação $(S B C)$. SBC, Porto Alegre - RS, $\mathrm{n}$.

[20] R. S. Rodrigues, W. L. Andrade, and L. M. R. S. Campos. 2016. Can Computational Thinking help me? A quantitative study of its effects on education. In 2016 IEEE Frontiers in Education Conference (FIE). IEEE, Eire, PA, USA, 1-8. https: //doi.org/10.1109/FIE.2016.7757409

[21] C. Rogers and M. Portsmore. 2004. Bringing Engineering to Elementary School In fournal of STEM Education : Innovations and Research; Auburn, Vol. 5. Semantic Scholar, USA, 17-28. https://search.proquest.com/docview/222795467? accountid $=146818$

[22] C. Schons, E. Primaz, and G. A. P. Wirth. 2004. Introdução a robótica educativa na instituição escolar para alunos do ensino fundamental da disciplina de língua espanhola através das novas tecnologias de aprendizagem. In Anais do I Workshop de Computação da Região Sul, Vol. 1. SBC, Porto Alegre - RS, 11.

[23] P. Seymour. 1986. LOGO: Computadores e Educação. Brasiliense (1986).

[24] I. M. L. Souza, W. L. Andrade, L. M. R Sampaio, and A. L. S. O Araujo. 2018. A Systematic Review on the use of LEGOtextsuperscript ${ }^{\circledR}$ Robotics in Education IEEE Frontiers in Education Conference (FIE) (2018), 1-9.

[25] I. M. L. Souza, R. S. Rodrigues, and W. L. Andrade. 2016. Explorando Robótica com Pensamento Computacional no Ensino Médio: Um estudo sobre seus efeitos na educação. In XXVII Brazilian Symposium on Computers in Education (Simpósio
Brasileiro de Informática na Educação - SBIE). SBC, Uberlândia- MG, 490-499.

[26] Charles Spearman. 2008. Spearman Rank Correlation Coefficient. In The Concise Encyclopedia of Statistics. Springer, New York, NY, 502-505. https://doi.org/10. 1007/978-0-387-32833-1 379

[27] J. M. Wing. 2006. Computational Thinking. Commun. ACM 49, 3 (March 2006), 33-35. https://doi.org/10.1145/1118178.1118215

[28] Education for Life ZOOM. 2010. ZOOM Education for Life, In Zoom for Education. Available in: hhttp://zoom.education, $\mathrm{n}$. 